\title{
Lamivudine versus Entecavir for Newly Diagnosed Hepatitis B Virus-Related Hepatocellular Carcinoma
}

Jung Hee Kim ${ }^{1}$, Dong Hyun Sinn ${ }^{1}$, Kyunga Kim², Hyeseung Kim², Geum-Youn Gwak ${ }^{1}$, Yong-Han Paik ${ }^{1}$, Moon Seok Choi ${ }^{1}$, Joon Hyeok Lee ${ }^{1}$, Kwang Cheol Koh ${ }^{1}$, and Seung Woon Paik ${ }^{1}$

${ }^{\prime}$ Department of Medicine, Samsung Medical Center, Sungkyunkwan University School of Medicine, and ${ }^{2}$ Biostatistics and Clinical Epidemiology Center, Samsung Medical Center, Seoul, Korea

\section{See editorial on page 869.}

Background/Aims: Antiviral therapy is a key component in the management of hepatitis B virus (HBV)-related hepatocellular carcinoma (HCC) patients. However, whether the potent drug entecavir is more effective than a less potent drug, such as lamivudine, in HBV-related HCC is not clear. Methods: A retrospective cohort of 451 newly diagnosed, HBV-related HCC patients without antiviral therapy at diagnosis, who started antiviral therapy with either entecavir $(n=249)$ or lamivudine $(n=202)$, were enrolled. Results: The median survival was longer for the entecavir group than for the lamivudine group, and lamivudine use (vs entecavir) was an independent factor for mortality (hazard ratio [HR], 1.49; $\mathrm{p}=0.002$ ). Lamivudine use (vs entecavir) was an independent risk factor for new-onset hepatic decompensation (HR, 1.67; $p=0.010$ ) in 318 patients without previous hepatic decompensation, and it was also an independent risk factor for recurrence after curative therapy (HR, 1.84; $p=0.002)$ in 117 patients who received curative therapy. The findings were similar in a propensity score-matched cohort. Conclusions: Overall survival, decompensation-free survival, and recurrence-free survival were better in the entecavir-treated patients than in the lamivudine treated-patients, indicating that the potent antiviral drug should be the preferred choice in HBV-related HCC patients. (Gut Liver 2016;10:939-947)

Key Words: Antiviral agents; Hepatitis B, chronic; Carcinoma, hepatocellular; Potency; Survival

\section{INTRODUCTION}

Chronic hepatitis B virus (HBV) infection is a major cause of hepatocellular carcinoma (HCC). ${ }^{1,2}$ Worldwide, approximately $54 \%$ of cases are attributed to HBV infection. ${ }^{3}$ Unfortunately, there is no cure for HBV; currently available treatments, such as interferons and nucleoside/nucleotide analogues (NUCs), can suppress viral replication but cannot eradicate the virus. ${ }^{4}$ However, NUCs are generally safe and well-tolerated oral medications, ${ }^{5}$ and NUC treatment has been shown to reduce the incidence of HCC, ${ }^{6-8}$ reduce the recurrence of HCC, ${ }^{9-11}$ and improve survival of patients diagnosed with HCC. ${ }^{10,12-14}$ Thus, NUC therapy has become an essential element in HBV-related HCC patients.

Although it is clear that HBV-related HCC patients should be treated with NUC, it is less clear what should be the firstline NUC in HBV-related HCC patients. Currently, there are several licensed NUCs, such as lamivudine, adefovir, telbivudine, clevudine, entecavir, and tenofovir, which can be used in HBV-related HCC patients. ${ }^{15-17}$ Long-term efficacy, safety, drug resistance, and cost are the major considerations in determining which NUC should be considered as first-line treatment. ${ }^{18}$ In patients without HCC, entecavir or tenofovir is recommended as a first-line NUC, as these two drugs have a significant advantage of low antiviral drug resistance over other drugs. ${ }^{15-17}$ However, in patients with HCC, there are no specific recommendations regarding the first-line NUC use, as there have only been few studies directly comparing the long-term efficacy between different types of NUCs in patients with HBV-related HCC. Entecavir is a potent drug that has shown superior virological and biochemical benefits as compared to lamivudine, ${ }^{19,20}$ and it has been found to be associated with a significantly lower risk of

Correspondence to: Dong Hyun Sinn

Department of Medicine, Samsung Medical Center, Sungkyunkwan University School of Medicine, 81 Irwon-ro, Gangnam-gu, Seoul 06351, Korea

Tel: +82-2-3410-3409, Fax: +82-2-3410-6983, E-mail: dh.sinn@samsung.com

Received on October 19, 2015. Revised on December 30, 2015. Accepted on January 6, 2016. Published online June 13, 2016 pISSN 1976-2283 eISSN 2005-1212 https://doi.org/10.5009/gnl15527

() This is an Open Access article distributed under the terms of the Creative Commons Attribution Non-Commercial License (http://creativecommons.org/licenses/by-nc/4.0) which permits unrestricted non-commercial use, distribution, and reproduction in any medium, provided the original work is properly cited. 
death or transplantation compared to lamivudine in NUC-naïve, non-HCC patients. ${ }^{21}$ However, entecavir is usually more costly than lamivudine; hence, lamivudine continues to be widely used, despite the additional costs incurred due to the development of drug resistance. ${ }^{22}$ Furthermore, lamivudine therapy has been shown to improve liver function, decrease HCC recurrence after curative therapy, and improve survival of patients with HBV-related HCC, ${ }^{23-25}$ and to the best of our knowledge, there are no randomized controlled trials that have demonstrated superior efficacy of a high potency drug over lamivudine in HBVrelated HCC patients.

It is known that the risk of HCC persists in patients treated with NUCs even when complete viral suppression is achieved. ${ }^{6}$ Lim et al. ${ }^{21}$ reported that HCC risk was comparable in patients who used entecavir or lamivudine. Kim et al. ${ }^{26}$ compared entecavir and telbivudine in HBV-related advanced HCC patients, and they reported that telbivudine is highly cost-effective and should be considered as a first-line antiviral agent in patients with advanced HCC. Shin et al. ${ }^{27}$ also compared lamivudine versus entecavir in patients with HBV-related advanced HCC, and they reported that lamivudine is sufficient and costeffective. Therefore, it is still an open question whether a potent drug should be used in patients with HBV-related HCC instead of lamivudine. Therefore, in this study, we compared overall survival, decompensation-free survival, and recurrence-free survival between patients who were treated with entecavir and lamivudine.

\section{MATERIALS AND METHODS}

\section{Study design, setting and participants}

This was a retrospective cohort study of HBV-related HCC patients. For this study, we used the HCC registry of Samsung Medical Center, which is a prospective registry that enrolls treatment naïve, newly-diagnosed HCC patients who received care at Samsung Medical Center, Seoul, Korea, from January 2005. When patients were newly diagnosed with HCC, welltrained abstractors collected the patient's data including age at diagnosis, gender, date of diagnosis, etiology, liver function (e.g., Child-Pugh class), tumor characteristics (e.g., number of tumors, maximal tumor size, presence and extent of portal vein invasion, and type of extrahepatic spread), tumor stage, and initial treatment modality, in a prospective manner. HCC was diagnosed either histologically or clinically according to the regional guideline. ${ }^{28}$ We screened a total of 3,514 patients who were registered in the HCC registry between January 1, 2005 and December 31, 2009. Among them, we excluded patients who met the following criteria to identify treatment naïve patients who started lamivudine or entecavir therapy after diagnosis of HCC (Supplementary Fig. 1): (1) patients with hepatitis B surface antigen negative; (2) patients who had coinfection with hepatitis $\mathrm{C}$ virus; (3) patients who did not use NUC during the follow-up period; and (4) patients who were using NUC at the time of HCC diagnosis. Then, we excluded patients who started therapy with NUC other than entecavir or lamivudine, and we also excluded patients who started entecavir or lamivudine after 30 days from the initial diagnosis of HCC. Finally, there were 451 antiviral treatment naïve, newly diagnosed HCC patients who started therapy with either entecavir or lamivudine within 30 days from the HCC diagnosis. The study protocol was reviewed and approved by the Institutional Review Board at Samsung Medical Center. Because the study was based on the retrospective analysis of existing administrative and clinical data, the requirement of obtaining informed patient consent was waived by the Institutional Review Board.

\section{End-outcome variables, definitions, and follow-up}

The primary end-outcome variable was overall survival. The secondary end-outcome variables were decompensation-free survival and recurrence-free survival. The initial date of diagnosis of HCC was considered as the baseline. All patients were followed up from the baseline till November 18, 2014. Patient survival data was collected from the National Statistics Service; therefore, all deaths at the time of survival assessment could be certified. Decompensation was defined by variceal bleeding, ascites, or hepatic encephalopathy, and it was defined in patients without history of prior decompensation. Recurrence was defined in patients who had received curative intent therapy, which included resection and radiofrequency ablation. For the decompensation-free survival and recurrence-free survival, patients were followed up from the baseline till the first episode of decompensation/recurrence or the last hospital visit.

\section{Variables}

We used the Samsung Medical Center HCC registry data for this study, which included age, gender, tumor stage, liver function, initial treatment methods, and so forth. For this study, we additionally collected data about the use of NUC, HBV DNA levels, new onset decompensation and recurrence. Serum HBV DNA levels were measured using hybrid capture assay and sandwich enzyme immunoassay (lower limit of detection $<20,000$ IU/mL; Digene Hybrid Capture II system; Digene Corp., Beltsville, MD, USA) before the year 2006, and then, they were replaced with quantitative real-time polymerase chain reaction (PCR) (COBAS TaqMan HBV DNA test; Roche, Branchburg, NJ, USA; lower limit of detection of $12 \mathrm{IU} / \mathrm{mL}$, which was lowered to $9 \mathrm{IU} / \mathrm{mL}$ in September 2009). We collected HBV DNA levels during the entire follow-up period, and complete virological response (CVR) was defined when HBV DNA became undetectable in serum during the follow-up period with either Digene Hybrid Capture II System or COBAS TaqMan HBV DNA test. We assessed the CVR rate at 1 year and during the entire followup period. Information on the use of other NUCs as a rescue therapy was also collected. 


\section{Statistical analysis}

Baseline characteristics were compared between the lamivudine versus entecavir treated patients using the Student $t$ test, Mann-Whitney U test, chi-square test, or Fisher exact test as appropriate. Overall survival, decompensation-free survival, and recurrence-free survival were compared between the lamivudine versus entecavir treated patients based on the Kaplan-Meier method using the log rank test. Cox proportional hazard regression analysis was performed to identify factors associated with each end-point. Multivariable model was constructed based on unadjusted analysis. Age and gender were included in the multi-

Table 1. Baseline Characteristics

\begin{tabular}{|c|c|c|c|c|c|c|}
\hline \multirow[b]{2}{*}{ Characteristic } & \multicolumn{3}{|c|}{ Overall $(\mathrm{n}=451)$} & \multicolumn{3}{|c|}{ Matched $(n=304)$} \\
\hline & $\begin{array}{l}\text { Entecavir } \\
(\mathrm{n}=249)\end{array}$ & $\begin{array}{l}\text { Lamivudine } \\
(\mathrm{n}=202)\end{array}$ & p-value & $\begin{array}{c}\text { Entecavir } \\
(\mathrm{n}=152)\end{array}$ & $\begin{array}{l}\text { Lamivudine } \\
(\mathrm{n}=152)\end{array}$ & p-value \\
\hline Age, yr & $51.8 \pm 8.8$ & $54.5 \pm 9.3$ & 0.001 & $52.9 \pm 7.6$ & $53.3 \pm 7.7$ & 0.78 \\
\hline Male & 209 (83.9) & $157(77.7)$ & 0.094 & $133(87.5)$ & $133(87.5)$ & 1.0 \\
\hline Child-Pugh score & $5(5-11)$ & $6(5-10)$ & 0.005 & $6(6-11)$ & $6(5-10)$ & 0.059 \\
\hline A & $200(80.3)$ & 145 (71.8) & & & & \\
\hline B & 39 (15.7) & $54(26.7)$ & & & & \\
\hline C & $10(4.0)$ & $3(1.5)$ & & & & \\
\hline Previous decompensation & $75(30.1)$ & $58(28.7)$ & 0.74 & $40(26.3)$ & $40(26.3)$ & 1.0 \\
\hline ECOG & $0(0-4)$ & $0(0-4)$ & 0.30 & & & \\
\hline 0 & 209 (83.9) & $162(80.2)$ & 0.54 & & & \\
\hline $1-4$ & $40(16.1)$ & 40 (19.8) & & & & \\
\hline mUICC stage & & & 0.56 & & & 0.91 \\
\hline I & $28(11.2)$ & $20(9.9)$ & & 16 (10.5) & $14(9.2)$ & \\
\hline II & $94(37.8)$ & $74(36.6)$ & & $63(41.4)$ & 60 (39.5) & \\
\hline III & 73 (29.3) & $71(35.1)$ & & $40(26.4)$ & $52(34.2)$ & \\
\hline IV & $54(21.7)$ & $37(18.4)$ & & $33(21.7)$ & $26(17.1)$ & \\
\hline Curative treatment & $110(44.2)$ & $67(33.2)$ & 0.017 & $60(39.5)$ & $60(39.5)$ & 1.0 \\
\hline Liver transplantation & 8 & 2 & & & & \\
\hline Surgical resection & 83 & 31 & & & & \\
\hline RFA & 19 & 34 & & & & \\
\hline HBV DNA level, $\log _{10} \mathrm{IU} / \mathrm{mL}$ & $5.7(1.2-8.0)$ & $5.5(1.3-8.0)$ & 0.31 & & & \\
\hline$<20,000 \mathrm{IU} / \mathrm{mL}$ & $45(18.1)$ & $49(24.4)$ & 0.10 & & & \\
\hline$\geq 20,000 \mathrm{IU} / \mathrm{mL}$ & 204 (81.9) & $152(75.6)$ & & & & \\
\hline Rescue therapy & $16(6.4)$ & $91(45.0)$ & $<0.001$ & & & \\
\hline \multicolumn{7}{|l|}{ Rescue methods } \\
\hline Adefovir add-on & 15 & 52 & & & & \\
\hline Switch to other drugs & 1 & 39 & & & & \\
\hline Response evaluation* & $192(77.1)$ & $138(68.3)$ & 0.75 & & & \\
\hline CVR within 1 year & $94 / 192(48.9)$ & $33 / 138(23.9)^{\dagger}$ & $<0.001$ & & & \\
\hline CVR during follow-up period & $139 / 192(72.3)$ & $92 / 138(66.7)^{\ddagger}$ & 0.052 & & & \\
\hline CVR without rescue therapy & 134 & 28 & & & & \\
\hline CVR with rescue therapy & 5 & 64 & & & & \\
\hline Time to CVR & $8.2(0.8-58.0)$ & $22.9(2.1-104.9)$ & $<0.001$ & & & \\
\hline
\end{tabular}

Data are presented as mean \pm SD, number $(\%)$, or median (range).

ECOG, Eastern Cooperative Oncology Group performance status; mUICC, modified Union for International Cancer Control; RFA, radiofrequency ablation; HBV, hepatitis B virus; CVR, complete virological response.

${ }^{*} \mathrm{HBV}$ DNA level was not followed up for 57 patients in the entecavir group and 64 patients in the lamivudine group. CVR was defined for undetectable HBV DNA levels; ${ }^{\dagger} 13$ and ${ }^{\ddagger} 30$ patients showed undetectable HBV DNA $(<20,000 \mathrm{IU} / \mathrm{mL})$ according to the Digene Hybrid Capture II system; otherwise, HBV DNA showed HBV DNA levels $(<12$ to $<9 \mathrm{IU} / \mathrm{mL})$ undetectable by real-time polymerase chain reaction. Patients were matched for curative treatment, previous decompensation, sex, mUICC stage, age and Child-Pugh score. 
variable model regardless of the p-value on unadjusted analysis. We also generated a propensity score matched cohort by matching variables between lamivudine and entecavir treated groups. The variable selected for propensity score matching were curative treatment, previous decompensation, sex, modified Union for International Cancer Control (mUICC) stage, age and ChildPugh score. Overall survival, decompensation-free survival, and recurrence-free survival were compared again between the lamivudine versus entecavir treated patients in a propensity-score matched cohort. All reported p-values were two-sided, and pvalue $<0.05$ was considered statistically significant.

\section{RESULTS}

\section{Baseline characteristics and antiviral response of NUC}

The baseline characteristics of the patients analyzed are shown in Table 1. Patients in the lamivudine group were older, and the median Child-Pugh score was higher. The two groups were not different in terms of previous decompensation history, Eastern Cooperative Oncology Group performance status (ECOG), and tumor stage (either Barcelona Clinic Liver Cancer staging system or mUICC). More number of patients in the entecavir group received curative treatment than those in the lamivudine group (Table 1). There was no difference in baseline HBV DNA levels between the two groups. However, rescue therapy was more frequently used in the lamivudine group. HBV DNA level was not followed in 57 patients of the entecavir group and in 64 patients of the lamivudine group; therefore, virological response could not be assessed in these patients. Among the evaluable patients, time to CVR was much shorter in the entecavir group. CVR rate within 1 year was also higher in the entecavir group and CVR was mostly achieved without rescue therapy, while this was not the case in the lamivudine group.

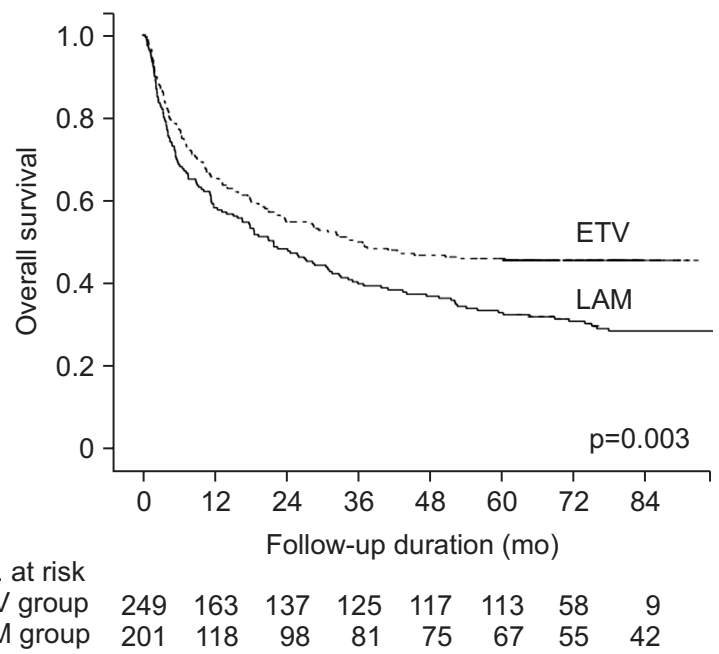

Fig. 1. Comparison of overall survival rate between entecavir (ETV)treated and lamivudine (LAM)-treated patients.

Table 2. Risk Factors for Mortality

\begin{tabular}{|c|c|c|c|c|c|c|c|c|}
\hline \multirow{2}{*}{ Characteristic } & \multicolumn{4}{|c|}{ Overall $(n=451)$} & \multicolumn{4}{|c|}{ Matched (n=304) } \\
\hline & $\begin{array}{l}\text { Unadjusted HR } \\
(95 \% \mathrm{CI})\end{array}$ & $\mathrm{p}$-value & $\begin{array}{c}\text { Multivariable HR } \\
(95 \% \mathrm{CI})\end{array}$ & $\mathrm{p}$-value & $\begin{array}{l}\text { Unadjusted HR } \\
\quad(95 \% \mathrm{CI})\end{array}$ & p-value & $\begin{array}{c}\text { Multivariable HR } \\
(95 \% \mathrm{CI})\end{array}$ & $\mathrm{p}$-value \\
\hline Age, /yr & $0.99(0.97-1.01)$ & 0.16 & $0.98(0.97-1.00)$ & 0.093 & $0.97(0.95-0.99)$ & 0.017 & $0.97(0.95-0.98)$ & 0.003 \\
\hline Male (vs female) & $1.36(0.99-1.87)$ & 0.055 & $1.69(1.21-2.35)$ & 0.002 & $1.40(0.88-2.23)$ & 0.15 & & \\
\hline Child-Pugh class B/C (vs A) & $2.25(1.74-2.90)$ & $<0.001$ & $1.24(0.93-1.66)$ & 0.14 & $2.36(1.73-3.21)$ & $<0.001$ & $1.23(0.85-1.77)$ & 0.26 \\
\hline \multicolumn{9}{|l|}{ Hepatic decompensation } \\
\hline None & 1 & & 1 & & 1 & & 1 & \\
\hline New-onset during follow-up & $2.25(1.65-3.06)$ & $<0.001$ & $1.44(1.05-1.98)$ & 0.023 & $2.06(1.42-2.97)$ & $<0.001$ & $1.42(0.97-2.08)$ & 0.070 \\
\hline Previous decompensation & $4.80(3.58-6.44)$ & $<0.001$ & $2.11(1.52-2.94)$ & $<0.001$ & $5.63(3.94-8.05)$ & $<0.001$ & $2.39(1.58-3.59)$ & $<0.001$ \\
\hline ECOG 1-4 (vs 0) & $2.79(2.12-3.67)$ & $<0.001$ & $1.46(1.10-1.95)$ & 0.009 & $2.77(1.98-3.87)$ & $<0.001$ & $1.46(1.01-2.10)$ & 0.041 \\
\hline \multicolumn{9}{|l|}{ mUICC stage } \\
\hline I & 1 & & 1 & & 1 & & 1 & \\
\hline II & $3.45(1.66-7.14)$ & 0.01 & $2.95(1.41-6.15)$ & 0.004 & $4.77(1.73-13.1)$ & 0.002 & $3.30(1.18-9.19)$ & 0.022 \\
\hline III & $7.37(3.59-15.1)$ & $<0.001$ & $4.19(2.02-8.70)$ & $<0.001$ & $8.28(3.01-22.7)$ & $<0.001$ & $4.11(1.47-11.5)$ & 0.007 \\
\hline IV & $20.1(9.66-41.6)$ & $<0.001$ & $9.51(4.49-20.1)$ & $<0.001$ & $30.1(10.8-83.9)$ & $<0.001$ & $12.6(4.42-35.9)$ & $<0.001$ \\
\hline Baseline HBV DNA, /log IU/mL & $1.03(0.92-1.08)$ & 0.93 & & & $1.00(0.96-1.04)$ & 0.94 & & \\
\hline HBV DNA $\geq 20,000 \mathrm{IU} / \mathrm{mL}$ (yes vs no) & $1.05(0.78-1.41)$ & 0.73 & & & $1.06(0.74-1.52)$ & 0.73 & & \\
\hline LAM (vs ETV) & $1.43(1.13-1.81)$ & 0.002 & $1.49(1.15-1.92)$ & 0.002 & $1.29(0.96-1.72)$ & 0.080 & $1.47(1.08-2.01)$ & 0.014 \\
\hline Curative treatment (vs palliative) & $0.14(0.10-0.19)$ & $<0.001$ & $0.24(0.17-0.34)$ & $<0.001$ & $0.13(0.09-0.20)$ & $<0.001$ & $0.22(0.14-0.35)$ & $<0.001$ \\
\hline
\end{tabular}

HR, hazard ratio; CI, confidence interval; ECOG, Eastern Cooperative Oncology Group performance status; mUICC, modified Union for International Cancer Control; HBV, hepatitis B virus; LAM, lamivudine; ETV, entecavir. 


\section{Factors associated with overall survival}

Median follow up duration of the enrolled patients was 28.6 months (minimum-maximum: 0.4-119.5 months). The risk factors for mortality are shown in Table 2. Lamivudine use (vs entecavir use) was an independent factor associated with mortality, along with male gender, new-onset or previous hepatic decompensation history, poor ECOG, mUICC stage, and curative treatment. The median survival was longer for the entecavir group than for the lamivudine group (36.8 months vs 21.9 months, $\mathrm{p}=0.002$ ). The 1-, 3-, and 5-year survival rates in the entecavir group were 65.4\%, 50.2\%, and 46.1\%, respectively, which were significantly higher than those in the lamivudine group (58.4\%, 40.1\%, and 33.1\% for the 1-, 3-, and 5-year survival rate, $\mathrm{p}=0.003$ ) (Fig. 1).

The impact of entecavir use (vs lamivudine use) according to the subgroup is summarized Supplementary Table 1 . When stratified by overall survival, there was no significant difference in median survival (4.0 months vs 3.8 months for the entecavir vs lamivudine groups, $\mathrm{p}=0.95$ ) among patients with mortality within 1 year, but survival was significantly better for the entecavir treated group among patients who survived more than a year (5-year survival rate, $70.5 \%$ vs $56.7 \%, p=0.004$ ). The 5-year survival rate was significantly different in mUICC stage I or II patients (70.4\% vs 54.2\%, p=0.007) or Child-Pugh class A patients (64.8\% vs $38.6 \%, \mathrm{p}=0.004)$, but it was not significantly different in mUICC stage III or IV patients $(22.8 \%$ vs $14.8 \%$, $\mathrm{p}=0.054)$ or Child-Pugh class B/C patients $(24.4 \%$ vs $19.3 \%$, $\mathrm{p}=0.99$ ) by NUC type (entecavir vs lamivudine). Survival difference was also noted in patients with baseline HBV DNA levels $\geq 20,000 \mathrm{IU} / \mathrm{mL}$ (5-year survival rate, $45.5 \%$ vs 30.9\%, $\mathrm{p}=0.004$ ), but not in patients with baseline HBV DNA levels $<20,000 \mathrm{IU} /$ $\mathrm{mL}(48.8 \%$ vs $40.0 \%, \mathrm{p}=0.23)$.

\section{Factors associated with decompensation-free survival and recurrence-free survival}

Among the 318 patients without previous hepatic decompensation, new-onset hepatic decompensation was noted in 117 patients (36.8\%). The decompensation-free survival rates were significantly better in the entecavir group than in the lamivudine group (84.7\%, 71.6\%, and $66.8 \%$ vs $76.4 \%$, 54.6\%, and $48.7 \%$ for the 1-, 3-, and 5-year decompensation-free survival

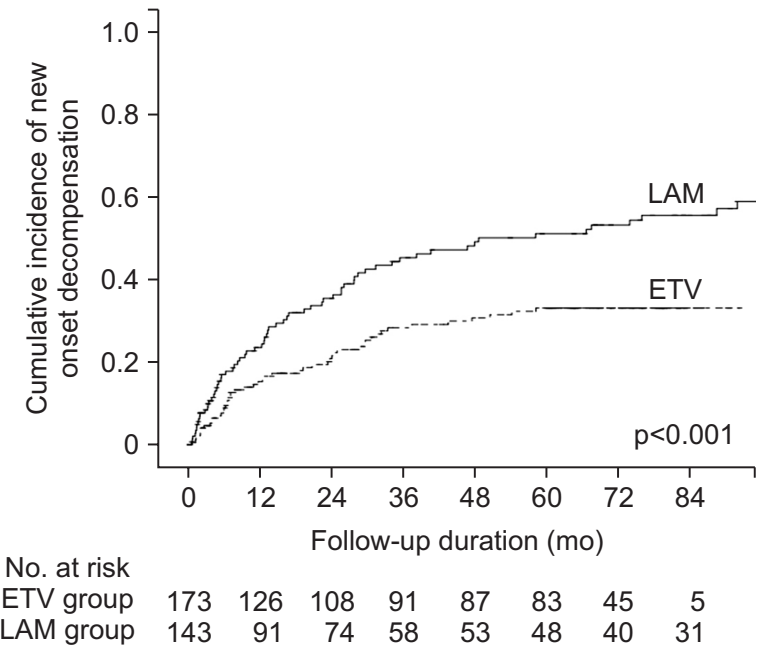

Fig. 2. Comparison of decompensation-free survival rate between entecavir (ETV)-treated and lamivudine (LAM)-treated patients.

Table 3. Risk Factors for New-Onset Hepatic Decompensation

\begin{tabular}{|c|c|c|c|c|c|c|c|c|}
\hline \multirow[b]{2}{*}{ Factor } & \multicolumn{4}{|c|}{ Overall $(n=318)$} & \multicolumn{4}{|c|}{ Matched ( $\mathrm{n}=224)$} \\
\hline & $\begin{array}{l}\text { Unadjusted HR } \\
\quad \text { (95\% CI) }\end{array}$ & $\mathrm{p}$-value & $\begin{array}{c}\text { Multivariable HR } \\
\quad(95 \% \mathrm{CI})\end{array}$ & $\mathrm{p}$-value & $\begin{array}{l}\text { Unadjusted HR } \\
(95 \% \mathrm{CI})\end{array}$ & p-value & $\begin{array}{c}\text { Multivariable HR } \\
\text { (95\% CI) }\end{array}$ & $\mathrm{p}$-value \\
\hline Age, /yr & $1.01(0.99-1.03)$ & 0.11 & $1.01(0.98-1.03)$ & 0.39 & $1.00(0.97-1.03)$ & 0.97 & $1.00(0.97-1.04)$ & 0.70 \\
\hline Male (vs female) & $0.89(0.57-1.38)$ & 0.60 & $0.86(0.54-1.39)$ & 0.56 & $0.93(0.50-1.71)$ & 0.81 & $0.96(0.50-1.81)$ & 0.90 \\
\hline Child-Pugh class B/C (vs A) & $1.29(0.72-2.30)$ & 0.38 & & & $0.96(0.44-2.08)$ & 0.92 & & \\
\hline ECOG 1-4 (vs 0) & $1.93(1.10-3.39)$ & 0.021 & $1.24(0.69-2.21)$ & 0.45 & $1.52(0.73-3.17)$ & 0.25 & & \\
\hline \multicolumn{9}{|l|}{ mUICC stage } \\
\hline I & 1 & & 1 & & 1 & & 1 & \\
\hline II & $1.10(0.60-2.02)$ & 0.73 & $0.94(0.51-1.73)$ & 0.84 & $1.34(0.62-2.90)$ & 0.44 & $0.91(0.41-2.02)$ & 0.83 \\
\hline III & $2.05(1.12-3.75)$ & 0.020 & $1.29(0.69-2.41)$ & 0.41 & $1.95(0.89-4.30)$ & 0.095 & $0.99(0.43-2.27)$ & 0.98 \\
\hline IV & $2.90(1.36-6.15)$ & 0.005 & $1.90(0.85-4.22)$ & 0.11 & $4.29(1.65-11.1)$ & 0.003 & $2.07(0.75-5.76)$ & 0.16 \\
\hline Baseline HBV DNA, /log IU/mL & $1.08(0.95-1.23)$ & 0.22 & & & $1.04(0.97-1.11)$ & 0.21 & & \\
\hline HBV DNA $\geq 20,000 \mathrm{IU} / \mathrm{mL}$ (yes vs no) & $1.69(0.98-2.91)$ & 0.056 & $1.84(1.05-3.21)$ & 0.031 & $1.67(0.90-3.08)$ & 0.10 & $1.65(0.87-3.11)$ & 0.12 \\
\hline LAM (vs ETV) & $1.90(1.31-2.76)$ & 0.001 & $1.67(1.12-2.48)$ & 0.010 & $1.39(0.89-2.16)$ & 0.14 & $1.45(0.92-2.29)$ & 0.10 \\
\hline Curative treatment (vs palliative) & $0.21(0.14-0.32)$ & $<0.001$ & $0.27(0.17-0.41)$ & $<0.001$ & $0.21(0.13-0.35)$ & $<0.001$ & $0.24(0.14-0.40)$ & $<0.001$ \\
\hline
\end{tabular}

HR, hazard ratio; CI, confidence interval; ECOG, Eastern Cooperative Oncology Group performance status; mUICC, modified Union for International Cancer Control; HBV, hepatitis B virus; LAM, lamivudine; ETV, entecavir. 
rate in the entecavir vs lamivudine groups, $\mathrm{p}<0.001$ ) (Fig. 2). Lamivudine use (vs entecavir) was an independent risk factor for new-onset hepatic decompensation, along with initial curative treatment and elevated HBV DNA levels at baseline (Table 3). Among the 177 patients who had received curative therapy, 117 patients (66.1\%) showed HCC recurrence. The 1-, 3-, and 5-year recurrence-free survival rates were better in the entecavir group (72.5\%, 60.6\%, and 52.3\%) than in the lamivudine group (56.7\%, 32.6\%, and 26.4\%, respectively, $\mathrm{p}=0.008$ ) (Fig. 3). Lamivudine use (vs entecavir) was an independent risk factor for recurrence after curative therapy, along with mUICC stage and elevated HBV DNA levels at baseline (Table 4).

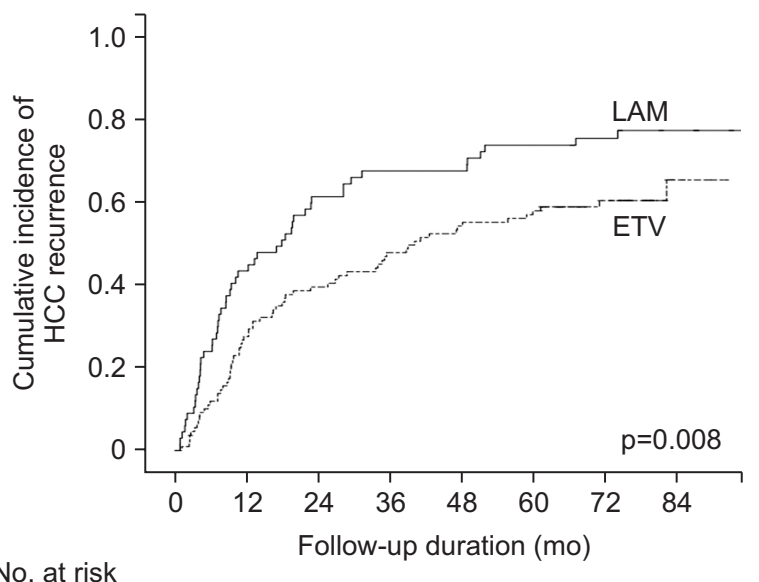

$\begin{array}{rrrrrrrrr}\text { ETV group } & 109 & 79 & 66 & 57 & 50 & 46 & 25 & 3 \\ \text { LAM group } & 66 & 38 & 26 & 21 & 21 & 17 & 13 & 10\end{array}$

Fig. 3. Comparison of recurrence-free survival rate between entecavir (ETV)-treated and lamivudine (LAM)-treated patients.

\section{Clinical outcome in a propensity score matched cohort}

After matching, there was no difference in age, gender, previous decompensation, mUICC stage and curative treatment between two groups. Child-Pugh score was slightly better in the lamivudine group (Table 1). In this matched cohort, lamivudine use (vs entecavir) was independent factor associated with overall mortality (Table 2), as well as recurrence after curative treatment (Table 4). The lamivudine use (vs entecavir) showed marginal association with new-onset hepatic decompensation (hazard ratio, 1.45; 95\% confidence interval, 0.92 to 2.29 ; $p=0.10$ ).

\section{DISCUSSION}

In this study, entecavir treated patients showed better overall survival, decompensation-free survival, and recurrence-free survival than lamivudine treated patients, and entecavir use was an independent factor associated with overall survival, decompensation-free survival, and recurrence-free survival. The baseline HBV DNA level was not different between the two groups; however, virological response was significantly better in entecavir treated patients (Table 1). The CVR rate for the entire followup period was not significantly different (72.3\% vs $66.7 \%$ for the entecavir vs lamivudine groups, $p=0.052$ ); however, a higher proportion of patients achieved a CVR at 1 year in the entecavir group (48.9\% vs 23.9\%, p<0.001), and many patients (64/92, $69.5 \%$ ) who started treatment with lamivudine required rescue therapy to achieve a CVR, while most of the patients (134/139, 96.4\%) in the entecavir group did not need rescue therapy to achieve a CVR. Time to CVR was also significantly longer in lamivudine treated patients compared to patients who started therapy with entecavir. As in NUC-naïve patients, suppression of viral activity by lamivudine was blunted by the development

Table 4. Risk Factors for Hepatocellular Carcinoma Recurrence after Curative Treatment

\begin{tabular}{|c|c|c|c|c|c|c|c|c|}
\hline \multirow[b]{2}{*}{ Factor } & \multicolumn{4}{|c|}{ Overall $(\mathrm{n}=177)$} & \multicolumn{4}{|c|}{ Matched $(n=120)$} \\
\hline & $\begin{array}{l}\text { Unadjusted HR } \\
\text { (95\% CI) }\end{array}$ & p-value & $\begin{array}{c}\text { Multivariable HR } \\
\quad(95 \% \mathrm{CI})\end{array}$ & p-value & $\begin{array}{l}\text { Unadjusted HR } \\
\quad(95 \% \mathrm{CI})\end{array}$ & $\mathrm{p}$-value & $\begin{array}{c}\text { Multivariable HR } \\
\quad(95 \% \mathrm{CI})\end{array}$ & p-value \\
\hline Age, /yr & $1.01(0.99-1.03)$ & 0.21 & $1.00(0.98-1.02)$ & 0.69 & $1.01(0.98-1.03)$ & 0.50 & $1.00(0.97-1.03)$ & 0.80 \\
\hline Male (vs female) & $1.38(0.84-2.26)$ & 0.19 & $1.17(0.70-1.95)$ & 0.54 & $1.06(0.53-2.13)$ & 0.85 & $0.96(0.47-1.96)$ & 0.92 \\
\hline Child-Pugh class B/C (vs A) & $1.10(0.59-2.06)$ & 0.74 & & & $0.98(0.45-2.14)$ & 0.97 & & \\
\hline Previous decompensation (vs no) & $1.28(0.69-2.38)$ & 0.43 & & & $1.72(0.69-4.26)$ & 0.24 & & \\
\hline ECOG 1-4 (vs 0) & $1.82(0.84-3.901)$ & 0.12 & & & $1.64(0.66-4.09)$ & 0.27 & & \\
\hline mUICC stage III/IV (vs I/II)* & $1.96(1.33-2.89)$ & 0.001 & $2.18(1.45-3.27)$ & $<0.001$ & $1.49(0.91-2.41)$ & 0.10 & $1.77(1.06-2.96)$ & 0.027 \\
\hline Baseline HBV DNA, /log IU/mL & $1.17(1.02-1.34)$ & 0.022 & & & $1.05(0.98-1.12)$ & 0.12 & & \\
\hline HBV DNA $\geq 20,000 \mathrm{IU} / \mathrm{mL}$ (yes vs no) & $1.53(0.93-2.50)$ & 0.091 & $1.87(1.12-3.10)$ & 0.015 & $1.32(0.76-2.28)$ & 0.32 & $1.74(0.98-3.08)$ & 0.057 \\
\hline LAM (vs ETV) & $1.63(1.13-2.36)$ & 0.008 & $1.84(1.25-2.72)$ & 0.002 & $1.96(1.25-3.05)$ & 0.003 & $2.18(1.38-3.46)$ & 0.001 \\
\hline
\end{tabular}

HR, hazard ratio; CI, confidence interval; ECOG, Eastern Cooperative Oncology Group performance status; mUICC, modified Union for International Cancer Control; HBV, hepatitis B virus; LAM, lamivudine; ETV, entecavir.

*Six patients with mUICC IVa who received resection were staged as mUICC IV based on multiple tumors, tumor size $>2 \mathrm{~cm}$ and segmental portal vein invasion. 
of resistance, for which rescue therapy was required in HBVrelated HCC patients. ${ }^{16}$

In this study, lamivudine use, elevated HBV DNA levels, and palliative treatment were independent risk factors for new-onset hepatic decompensation. Our findings are in line with previous studies that reported poor outcome in patients with high baseline HBV DNA levels, ${ }^{29}$ and improved liver function by NUC therapy in HBV-related HCC patients. ${ }^{30}$ Notably, new-onset hepatic decompensation was an independent factor for overall survival. This study showed that a higher potency antiviral agent is better than a less potent drug for preventing hepatic decompensation, which may translate into a better overall survival. We also noted that lamivudine use was an independent risk factor for recurrence after initial curative treatment, together with advanced tumor stage and elevated HBV DNA levels. In a meta-analysis, NUC therapy was associated with recurrencefree survival and overall survival in HCC patients after curative treatment. This study provides information that a potent antiviral agent is better than a less potent drug for preventing recurrence after curative therapy. These findings strongly indicate that the potent antiviral drug should be the preferred choice in HBV-related HCC patients.

In a previous study, a less potent drug was reported to be equally effective as entecavir in advanced HCC patients. ${ }^{26,27}$ Shin et al. ${ }^{27}$ compared 87 patients treated with lamivudine to 47 patients treated with entecavir, and they reported that the virological response and overall survival were similar. Kim et al. ${ }^{26}$ compared 39 patients treated with telbivudine to 47 patients treated with entecavir, and they also reported that the virological response and survival were similar. In this study, when we divided the patients according to the tumor stage, the 5-year overall survival rate was significantly different in mUICC stage I or II patients $(70.4 \%$ vs $54.2 \%$ for entecavir vs lamivudine groups, $p=0.007$ ), but this difference was marginal in patients with mUICC stage III or IV tumor $(22.8 \%$ vs $14.8 \%, p=0.054)$. As NUC does not have any antitumor effect, ${ }^{16}$ the efficacy of lamivudine or entecavir heavily depends on suppression of viral activity. Efficacy of lamivudine will be worse than that of entecavir when patients experience viral flares consequent to the development of lamivudine resistance. As it takes time to develop lamivudine resistance, the type of antiviral therapy may not have a significant impact on overall survival in patients with poor survival. In this study, survival advantage of entecavir was not observed in the subgroup with advanced HCC, poor liver function (e.g., Child-Pugh Class B/C), previous hepatic decompensation, mortality within a year, and those with low baseline HBV DNA levels. The type of antiviral therapy may have minimal impact in patient survival in some subgroups (e.g., advanced HCCs with poor expected survival, low baseline HBV DNA levels), and lamivudine use might be a cost-effective approach in those patients. However, accurate prediction of survival or virological response is sometimes very challeng- ing, which raises the question whether selecting antiviral drugs based on the expected survival or the expected antiviral resistance can be a reasonable approach. Therefore, although lamivudine may be a cost-effective approach in some selected patients, the preferred choice in HBV-related HCC patients should be a potent antiviral drug as in non-HCC patients.

There are some limitations to this study, which warrants careful interpretation of the results. First, this study is not a randomized controlled trial. Thus, our findings are potentially subject to selection bias and confounding. We tried to balance several important baseline factors that are associated with overall, decompensation-free and recurrence-free survival by propensity score matching. The findings were similar in a propensity score matched cohort, indicating our findings are true. However, this approach also has limitations, as all the important variables cannot be exactly matched. Second, as NUC does not have any antitumor effect, the response to antiviral therapy in achieving CVR could be more important factor predicting overall survival, decompensation-free survival, and recurrence-free survival than the drug type. We could not use CVR in this study, as many patients have not been followed-up for their HBV DNA levels after AVT (22.8\% in entecavir group and 31.6\% in lamivudine group). Also, the methods of HBV DNA measurement changed over time; hence, the lower limit of detection was higher for patients in the lamivudine group. Among the 33 patients in the lamivudine group who had undetectable HBV DNA levels, 13 patients had undetectable HBV DNA levels with Digene Hybrid Capture II system, which means that some of them may show detectable HBV DNA levels when the more sensitive real-time PCR assay was applied. Time to CVR also heavily depends on HBV DNA measurement methods. Third, date of diagnosis was significantly earlier in the lamivudine group. The lamivudine group included patients diagnosed between 2005 and 2007, and the entecavir group included patients diagnosed between 2007 and 2009. The discrepancy in treatment period may correlate with technical improvement of treatment modality in the entecavir group, which may have caused a higher rate of curative treatment in the entecavir group (44.2\% vs 33.2\%, $\mathrm{p}=0.017$ ), without a difference in the mUICC stage, which may have provided a survival advantage in the entecavir group. Therefore, to obtain stronger evidence, a randomized controlled trial is necessary; however, our observation data suggest that performing a randomized controlled trial might be deemed unethical, given the proven superior efficacy of entecavir in achieving a virological response without rescue therapy during follow-up. In this respect, this study may help guide physicians in selecting first-line NUCs in newly diagnosed HBV-related HCC patients.

The NUCs are an essential component of the management of HBV-related HCC patients. Our data suggest that a potent antiviral drug should be the preferred choice in HBV-related HCC patients, given the superior overall survival, decompensationfree survival, and recurrence-free survival in entecavir treated 
patients over lamivudine treated patients. The survival benefit of choosing a highly potent drug over a less potent drug will be higher when patient survival is long enough. A less potent drug may be an option in patients with low expected survival, especially in a resource-limited setting, but a highly potent drug should be the preferred choice in the HBV-related HCC patients.

\section{CONFLICTS OF INTEREST}

No potential conflict of interest relevant to this article was reported.

\section{REFERENCES}

1. Beasley RP. Hepatitis B virus. The major etiology of hepatocellular carcinoma. Cancer 1988;61:1942-1956.

2. Perz JF, Armstrong GL, Farrington LA, Hutin YJ, Bell BP. The contributions of hepatitis $B$ virus and hepatitis $C$ virus infections to cirrhosis and primary liver cancer worldwide. J Hepatol 2006;45: 529-538.

3. Yang JD, Roberts LR. Hepatocellular carcinoma: a global view. Nat Rev Gastroenterol Hepatol 2010;7:448-458.

4. Trépo C, Chan HL, Lok A. Hepatitis B virus infection. Lancet 2014; 384:2053-2063.

5. Fontana RJ. Side effects of long-term oral antiviral therapy for hepatitis B. Hepatology 2009;49(5 Suppl):S185-S195.

6. Yam JC, Chong GS, Wu PK, Wong US, Chan CW, Ko ST. Predictive factors affecting the short term and long term exodrift in patients with intermittent exotropia after bilateral rectus muscle recession and its effect on surgical outcome. Biomed Res Int 2014;2014:482093.

7. Liaw YF, Sung JJ, Chow WC, et al. Lamivudine for patients with chronic hepatitis B and advanced liver disease. N Engl J Med 2004;351:1521-1531.

8. Wu CY, Lin JT, Ho HJ, et al. Association of nucleos(t)ide analogue therapy with reduced risk of hepatocellular carcinoma in patients with chronic hepatitis B: a nationwide cohort study. Gastroenterology 2014;147:143-151.e5.

9. Wu CY, Chen YJ, Ho HJ, et al. Association between nucleoside analogues and risk of hepatitis B virus-related hepatocellular carcinoma recurrence following liver resection. JAMA 2012;308:19061914.

10. Sun P, Dong X, Cheng X, Hu Q, Zheng Q. Nucleot(s)ide analogues for hepatitis B virus-related hepatocellular carcinoma after curative treatment: a systematic review and meta-analysis. PLoS One 2014;9:e102761.

11. Sohn W, Paik YH, Kim JM, et al. HBV DNA and HBsAg levels as risk predictors of early and late recurrence after curative resection of HBV-related hepatocellular carcinoma. Ann Surg Oncol 2014;21:2429-2435.

12. Sohn W, Paik YH, Cho JY, et al. Sorafenib therapy for hepatocellular carcinoma with extrahepatic spread: treatment outcome and prognostic factors. J Hepatol 2015;62:1112-1121.

13. Huang G, Lau WY, Wang ZG, et al. Antiviral therapy improves postoperative survival in patients with hepatocellular carcinoma: a randomized controlled trial. Ann Surg 2015;261:56-66.

14. Hann HW, Coben R, Brown D, et al. A long-term study of the effects of antiviral therapy on survival of patients with HBVassociated hepatocellular carcinoma (HCC) following local tumor ablation. Cancer Med 2014;3:390-396.

15. European Association for the Study of the Liver. EASL clinical practice guidelines: management of chronic hepatitis B virus infection. J Hepatol 2012;57:167-185.

16. Korean Association for the Study of the Liver. KASL clinical practice guidelines: management of chronic hepatitis B. Clin Mol Hepatol 2012;18:109-162.

17. Lok AS, McMahon BJ. Chronic hepatitis B: update 2009. Hepatology 2009;50:661-662.

18. Yapali S, Lok AS. Potential benefit of telbivudine on renal function does not outweigh its high rate of antiviral drug resistance and other adverse effects. Gastroenterology 2014;146:15-19.

19. Chang TT, Gish RG, de Man R, et al. A comparison of entecavir and lamivudine for HBeAg-positive chronic hepatitis B. N Engl J Med 2006;354:1001-1010.

20. Lai CL, Shouval D, Lok AS, et al. Entecavir versus lamivudine for patients with HBeAg-negative chronic hepatitis B. N Engl J Med 2006;354:1011-1020.

21. Lim YS, Han S, Heo NY, Shim JH, Lee HC, Suh DJ. Mortality, liver transplantation, and hepatocellular carcinoma among patients with chronic hepatitis B treated with entecavir vs lamivudine. Gastroenterology 2014;147:152-161.

22. World Health Organization (WHO). Guidelines for the prevention, care and treatment of persons with chronic hepatitis B infection [Internet]. Geneva: WHO; c2015 [cited 2015 Apr 21]. Available from: http://www.who.int/hiv/pub/hepatitis/hepatitis-b-guidelines/en/.

23. Xu X, Huang $\mathrm{P}$, Tian $\mathrm{H}$, et al. Role of lamivudine with transarterial chemoembolization in the survival of patients with hepatocellular carcinoma. J Gastroenterol Hepatol 2014;29:1273-1278.

24. Yin J, Li N, Han Y, et al. Effect of antiviral treatment with nucleotide/nucleoside analogs on postoperative prognosis of hepatitis $B$ virus-related hepatocellular carcinoma: a two-stage longitudinal clinical study. J Clin Oncol 2013;31:3647-3655.

25. Kuzuya T, Katano Y, Kumada T, et al. Efficacy of antiviral therapy with lamivudine after initial treatment for hepatitis B virus-related hepatocellular carcinoma. J Gastroenterol Hepatol 2007;22:19291935.

26. Kim YW, Kwon JH, Chung E, et al. Short term virologic efficacies of telbivudine versus entecavir against hepatitis B-related hepatocellular carcinoma. Gastroenterol Res Pract. 2015;2015:181065.

27. Shin HS, Kim SU, Park JY, et al. Antiviral efficacy of lamivudine versus entecavir in patients with hepatitis B virus-related advanced hepatocellular carcinoma. J Gastroenterol Hepatol 2012;27:15281534. 
Kim JH, et al: Antiviral Agents and Newly Diagnosed HBV Related HCC 947

28. Korean Liver Cancer Study Group; National Cancer Center, Korea. Practice guidelines for management of hepatocellular carcinoma 2009. Korean J Hepatol 2009;15:391-423.

29. Yu SJ, Kim YJ. Hepatitis B viral load affects prognosis of hepato- cellular carcinoma. World J Gastroenterol 2014;20:12039-12044.

30. Jin YJ, Shim JH, Lee HC, et al. Suppressive effects of entecavir on hepatitis B virus and hepatocellular carcinoma. J Gastroenterol Hepatol 2011;26:1380-1388. 